\title{
THE INTERSECTION OF INDECOMPOSABLE CONTINUA
}

\author{
JOHN JOBE
}

The theme of this paper is to reveal a fundamental result concerning the 2-finished sum of compact continua, one of which is hereditarily indecomposable.

Definition. The set $M$ is the $k$-finished sum of a set of subcontinua, $\left\{M_{1}, \cdots, M_{k}\right\}$, if and only if

$$
M=\bigcup_{i=1}^{k} M_{i}
$$

and for each fixed $j, 1 \leqq j \leqq k$,

$$
M_{j}-\bigcup_{1 \leq i \leq k ; i \neq j} M_{i} \neq \varnothing .
$$

In this paper we shall consider the space $S$ to be a Moore space satisfying Axiom 0 and Axiom 1 of R. L. Moore. The boundary of a set $D$ with respect to a set $M$ will be denoted by $F(D)_{M}$.

THEOREM. If $M$ is the 2-finished sum of compact continua, $M_{1}$ and $M_{2}$, such that $M_{1}$ is hereditarily indecomposable and $M_{1} \cap M_{2} \neq \varnothing$, then there exists at least one point in $M_{1} \cap M_{2}$ which is a limit point of both $\left(M_{1}-M_{2}\right)$ and $\left(M_{2}-M_{1}\right)$.

Proof. Since $M_{1} \cap M_{2} \neq \varnothing$, then $M$ is a compact continuum. Suppose that no point of $M_{1} \cap M_{2}$ is a limit point of both $H=M_{1}-M_{2}$ and $K=M_{2}-M_{1}$. That is, suppose $F(H)_{M} \cap F(K)_{M}=\varnothing$. The supposition implies that $\bar{H} \cap \bar{K}=\varnothing$.

Let $T$ be a component of $H$. The reference [1] implies that there exists a point $p \in F(H)_{M} \cap \bar{T}$. Also it is noted that $\bar{T} \subset M_{1}$ since $M_{1}$ is a closed point set containing $T$. Since $H$ is a domain relative to $M$ then $p \notin H$ and $p \in M_{2}$.

The point $p \notin \bar{K}$ since $p \in M_{1} \cap F(H)_{M}$. Thus the point set $\left(M_{2}-\bar{K}\right)$ $=M_{2} \cap(M-\bar{K})$ is a domain relative to $M_{2}$ containing $p$. Let $L$ be the component of $\left(M_{2}-\bar{K}\right)$ containing the point $p$. The reference [1] implies that there exists a point $q \in F(K)_{M} \cap \bar{L}$. The point $q \notin \bar{T}$ for if so then $q \in F(H)_{M}$ which contradicts the supposition. The point set $\bar{L} \subset M_{1}$ since $M_{1}$ is a closed point set containing $L$.

By the definition of both $\bar{T}$ and $\bar{L}$ we know that each is a subcontinuum of $M_{1}$. Then since $p \in \bar{T} \cap \bar{L}$ the point set $\bar{T} \cup \bar{L}$ is a sub-

Received by the editors October 25, 1968. 
continuum of $M_{1}$. Since $q \notin \bar{T}$ then $\bar{L}-\bar{T} \neq \varnothing$. Also since $T \subset H$ and $\bar{L} \cap H=\varnothing$, then $\bar{T}-\bar{L} \neq \varnothing$. Thus the point set $\bar{T} \cup \bar{L}$ is a decomposable subcontinuum of $M_{1}$. This contradicts $M_{1}$ being hereditarily indecomposable, and thus the theorem is proved.

\section{BIBLIOGRAPHY}

1. R. L. Moore, Foundations of point set theory, Amer. Math. Soc., Colloq. Publ., vol. 13, Amer. Math. Soc., Providence, R. I., 1962.

Oklahoma State University 